\title{
Korean university life in a network perspective: Dynamics of a large affiliation network
}

\author{
Petter Holme \\ Department of Physics, University of Michigan, Ann Arbor, MI 48109 \\ Sung Min Park and Beom Jun Kim \\ Department of Molecular Science and Technology, Ajou University, Suwon 442-749, Korea \\ Christofer R. Edling \\ Department of Sociology, Stockholm University, 10691 Stockholm, Sweden
}

\begin{abstract}
We investigate course registration data of 18 semesters at a Korean University to portray the time evolution of students' positions in the network of fellow students. Apart from being a study of the social positions of students, the present work is also an example of how large-scale, time resolved, affiliation networks can be analyzed. For example we discuss the proper definitions of weights, and propose a redefined weighted clustering coefficient. Among other things, we find that the students enter the network at the center and are gradually diffusing towards the periphery. On the other hand, the ties to the classmates of the first semester (still present at the university) will, on average, become stronger as time progresses.
\end{abstract}

\section{INTRODUCTION}

Networks constitute, along differential equation models and cellular automata, a fundamental framework for analyzing and modeling complex systems $(1 ; 6 ; 17)$. The advent of modern database technology has greatly vitalized the statistical study of networks. Networks of electronic communication, genetic interaction, hyperlinked web-pages, and so on, are available in sizes up to hundreds of millions of vertices (5), to be compared with the data sets of a decade, or so, ago that mostly were curated manually edge by edge. The sizes of these computer compiled data sets put new demands on algorithms and analysis methods - an $O\left(N^{5}\right)$ algorithm, where $N$ is the number of vertices, may work perfectly to analyze a food-web (14) but would be intractable for analysis of the contacts in an Internet community (10). On the other hand, larger sizes rids the data of finite size biases, and allows one to extrapolate the conclusions to the large size limit. These new available data sets have created a new sub-field of network-sociology (24) and are the main reasons statistical physicists (traditionally working in the large size limit) have been joining this interdisciplinary field.

In the present paper we use course registration data from the mid-size Korean University, Ajou University, located in Suwon, Republic of Korea. Our data set consists of lists of undergraduate students registered to courses for 18 semesters (two semesters per year), starting with the spring semester 1996 and ending with the fall semester 2004. The basic network of a particular semester is an "affiliation network" where students and courses are two separate sets of vertices and edges link students to courses to which they are registered. From such a "two-mode" network (a network with two classes of vertices) one can make a "one-mode" projection to the set of students (or courses), where one student (course) is connected to another if they have a link to the same course (student) in the affiliation network. In this paper we only consider projections to the set of students and try to answer how a student's time at the university can be characterized by network statistics. Some basic statistics of the network of the fall semester 2003 is given in (19). Apart from allowing us to view Korean students' time at the university, and maybe university students in general, we introduce new structural quantities and methods of analyzing time resolved affiliation network data.

\section{CONSTRUCTION OF THE NETWORKS}

From our lists of course-registration data we would like to construct networks where an edge between two persons means that these two students are likely to be acquainted. We will consider both weighted and unweighted networks. For the weighted networks, we want a high weight to represent a high probability of the two students being acquainted. If two students take a course with few attendees they are more likely to know each other than if the course is large. Following Ref. (16) we let a specific course $m$ contribute to the weight between two students taking it with the inverse of the total number of students taking $m, 1 / n(m)$. But if two students take many courses in common, the chance of them being acquainted is larger. To account for this we just sum the contribution of each individual course

$$
w(i, j)=\sum_{m \in C} \frac{\delta_{m}(i, j)}{n(m)},
$$

where $C$ is the set of all courses, $\delta_{m}(i, j)$ is unity if both $i$ and $j$ take $m$ and $i \neq j$, and zero otherwise. Now, as mentioned, our data is time resolved, which gives us a possibility of accounting for the cessation of acquaintances - two persons are less likely to feel acquainted if they took a course together three years ago, than if they were in the same class the last semester. The weight at a given time $t$ can thus be written as

$$
w(i, j, t)=\sum_{t^{\prime} \leq t} \sum_{m \in C} \frac{\rho\left(t-t^{\prime}\right) \delta_{m}\left(i, j, t^{\prime}\right)}{n\left(m, t^{\prime}\right)},
$$



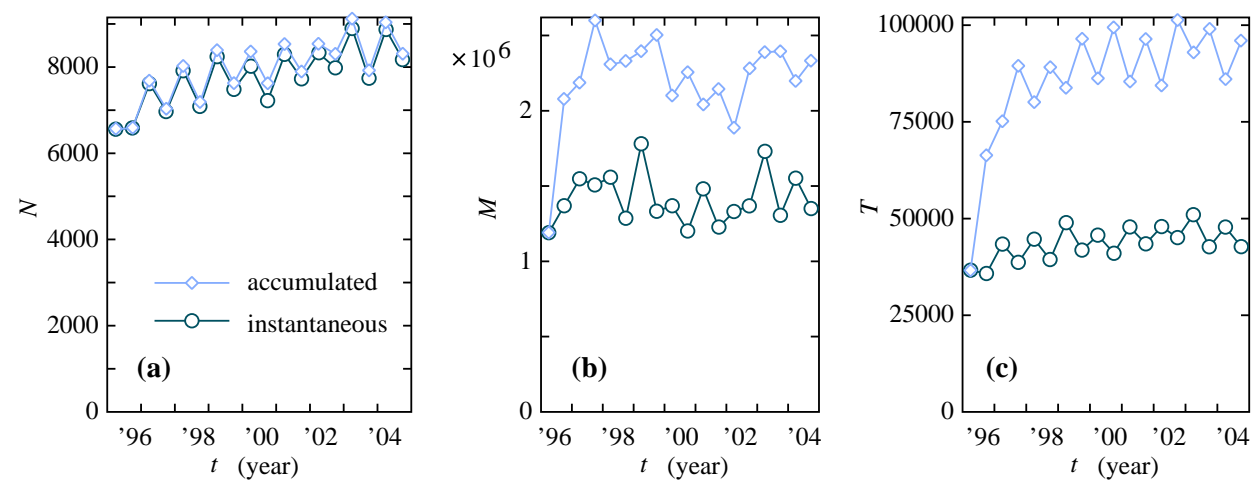

FIG. 1 The number of vertices $N$ (a), edges $M$ (b) and the total weight $T$ (c) in the networks. The instantaneous networks are the giant components of the projection of the course-student network for a given semester onto the set of students. The accumulated networks are giant components of the one-mode projection of accumulated course-student networks onto the sets of currently active students. An accumulated course-student network contains all courses in the data set given the specified semester or earlier.
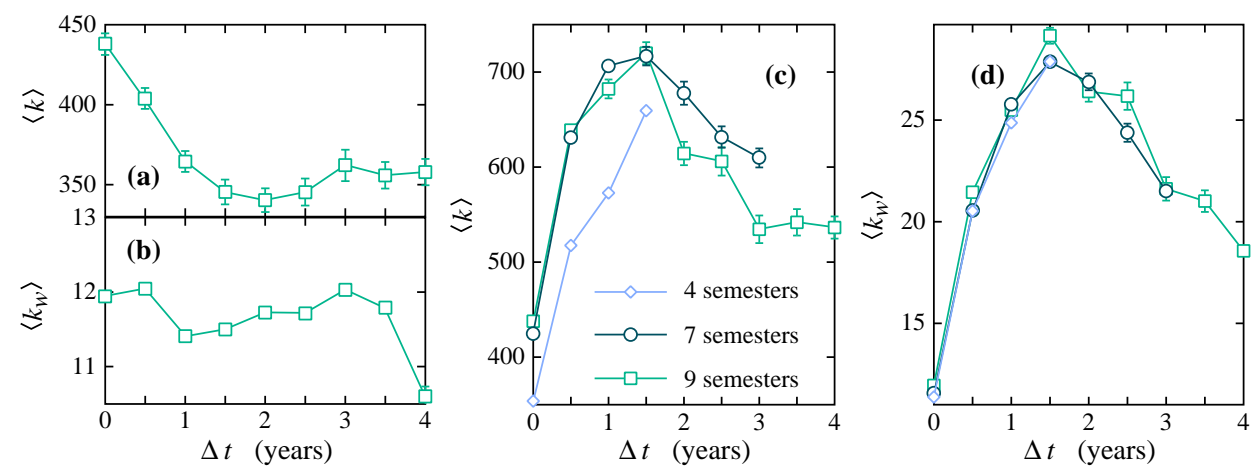

FIG. 2 The average degrees for students studying four, seven, and nine semesters. (a) and (b) show the unweighted and weighted results respectively for instantaneous networks. (c) and (d) show the unweighted and weighted results respectively for accumulated networks.

where $\rho(t)$ is a non-increasing function that accounts for the decay of friendships over time $(9)$, and $n(m, t)$ is the number of students taking the course $m$ at the semester $t$, and $\delta_{m}(i, j, t)$ is the corresponding generalization of $\delta_{m}(i, j)$. We will use the two simplest decay functions: Either we let $\rho(t)$ decay as fast as possible and thus be unity for $t=0$ and zero for $t>0$ giving an instantaneous network, or we let $\rho(t)$ not decay at all (be constantly unity) and obtain an accumulated network.

Some of the quantities we will use are based on shortest path lengths. Since these are not defined in a general network we reduce our graphs to their giant components (largest connected subgraphs). In these, the numbers of vertices are 95-100\% of the original networks. Another technicality concerns the temporal boundaries-we do not know how long a student of the first semester of our data has been at the university, and we do not know how long a student present the last semester will stay. We handle this problem by not including the students present the first and last semester in the averages of our quantities. There are, of course, students who are on leave from the university a few semesters. We estimate around $\sim 15 \%$ of the data points are students who have returned from a break. How to treat these students is a dilemma: On the one hand, one would like the number of active semesters to be the measure of time (in studying the time evolution of quantities). On the other hand, one cannot exclude the returning students from the network, after all they are a part of the network after their return. We will choose the latter alternative, and treat a student on leave as still present at the university, but with zero degree. The sizes of the networks are presented in Fig. 1] We note that the accumulated networks sometimes has a higher $N$ than the instantaneous counterparts. This is because the additional edges of the accumulated networks can give a larger giant component - the number of edges in the projection to the set of students is the same. The jagged shape of Fig. 11a) is an effect of that more students enter the university the spring semester $(\sim 1957$ on average $)$ than the fall semester $(\sim 353$ on average). Assuming the half of students stay an odd (or even) number of semesters the spring semesters would expect a difference of $(1957-353) / 2 \approx 802$ between the spring and fall semesters. That the actual average difference is smaller ( 635 on average), is an effect of that more students take an even number of semesters since many programs is for an even number of semesters.

\section{THE MEASURED STATISTICS}

In this section we will present the quantities we measure, the values we obtain and interpret these. All our curves are functions of the time (number of semesters) $\Delta t$ a student has 
studied at the university. To avoid, as much as possible, that behavioral differences get averaged away, we measure the curves for students present at the university a fixed time. As it turns out, these curves are qualitatively similar (but sometimes differs quantitatively).

\section{A. Degree and weight}

A fundamental vertex-quantity of unweighted networks is the degree, $k(i)$, defined as the number of edges leading to a vertex $i$. Degree gives a measure of how central a vertex is in its local surrounding (and is therefore sometimes referred to as degree centrality (22)). The straightforward generalization of degree to weighted networks is (22):

$$
k_{w}(i)=\sum_{j} w(i, j) .
$$

In Figs. 2(a) and (b) the degree, in its unweighted and weighted versions, for instantaneous networks is plotted for students staying nine semesters at the University. Other lengths of study give the same general shape of both $\langle k\rangle$ and $\left\langle k_{w}\right\rangle$-an early decrease of $\langle k\rangle$ that flattens out, and a rather constant $\left\langle k_{w}\right\rangle$. This decrease is probably a result of students taking increasingly specialized courses, in smaller and smaller classes. Results for accumulated networks are displayed in Figs. 2/c) and (d). These have maximum around the fourth semester. Note that if none of student A's fellow students of the first semester leave before A, then A's degree would be strictly increasing in the accumulated networks. The decreasing part is a result of old neighbors (vertices one edge away) exiting the network.

\section{B. Relation to fellow freshmen}

To get a picture of how the ties come and go in our networks, we study the relation of a student to her, or his, fellow freshmen (defined as the neighborhood of a student the first semester at the university). In Fig. 3 a) we display the average fraction of fellow freshmen remaining after $t$ semesters $\phi$ for students who stay four, seven, and nine semesters at the university. $\phi$ is exceptionally similar for number of semesters at the university.

Students coming and leaving the University is of course not the only dynamic mechanism present. The choice of courses can make students drift apart or get closer. This can be measured by graph distances. The distance of a path $P$ between two students, in the unweighted network, is the number of edges in it, whereas for the weighted network it is the sum of reciprocal weights $(16)$ :

$$
\begin{aligned}
d(i, j) & =\min _{P \in \mathcal{P}(i, j)}|P| \\
d_{w}(i, j) & =\min _{P \in \mathcal{P}(i, j)} \sum_{\left(i^{\prime}, j^{\prime}\right) \in P} \frac{1}{w\left(i^{\prime}, j^{\prime}\right)},
\end{aligned}
$$

where $\mathcal{P}(i, j)$ is the set of paths between $i$ and $j$. In Fig. 3 b) and (c) we plot the average shortest distances between a nine-

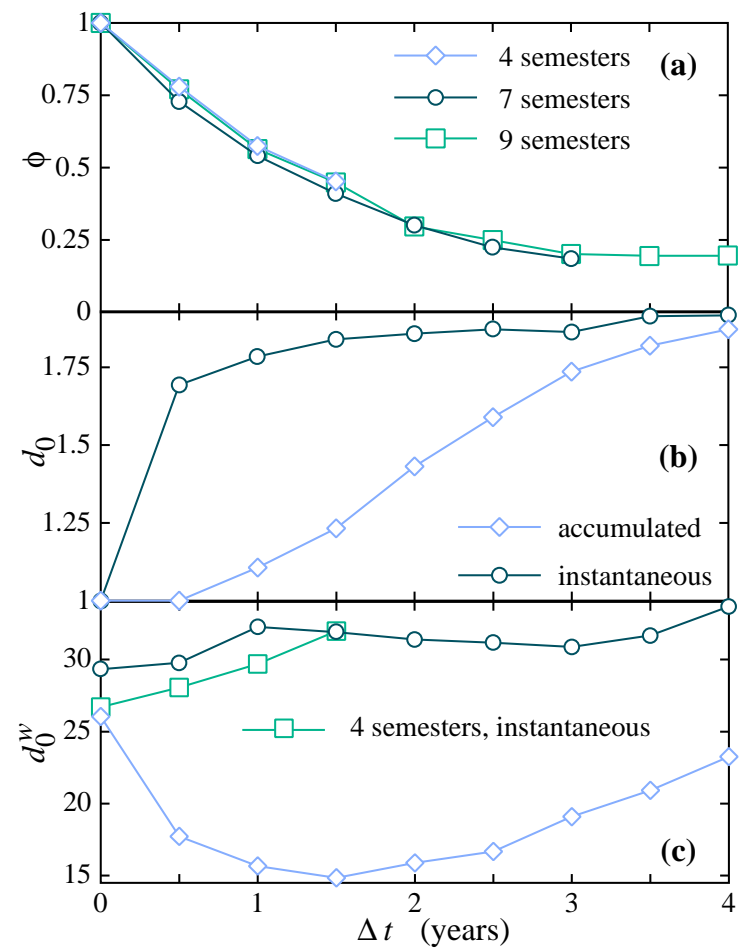

FIG. 3 Statistics about the fellow students of the first semester. (a) shows the fraction $\phi$ of students of the first semester that still are students of the university. (b) and (c) display the distance to the neighborhood of the first semester of students staying nine semesters in total (unless otherwise stated). (b) shows the unweighted version $d_{0}$ and (c) shows the weighted counterpart $d_{0}^{w}$. All errorbars are smaller than the symbol size.

semester student and her, or his, fellow freshmen still present in the network for unweighted $d_{0}$ and weighted $d_{0}^{w}$ distances respectively. The unweighted distances are strictly increasing. So one's fellow students of the first semester does not only become fewer in number but also further away in distances of binary networks. In the authors' personal experience, the acquaintances with some of the fellow freshmen grew stronger with time. This phenomenon is visible for the weighted distances of Fig. 3] c). Interestingly, $d_{0}^{w}$ of the instantaneous network has both a maximum and a minimum in the interior of the $\Delta t$ interval. We believe that this is because the students choosing different courses than the nine-semester students (and thus causing the early growth of $d_{0}^{w}$ ) are often leaving the university sooner than the nine semester students. This picture is supported by the monotonous growth seen for four-semester students. To calculate weighted distances for all vertices is the computationally most demanding part of our analysis, requiring $O(M N \log (N))$ time with a Dijkstra's algorithm implemented using a binary heap for storing the subsequently shortest distances (16).

\section{Closeness centralities}

In Sect. III.A we discussed the behavior of degree, a quantity measuring the centrality of a student in the local surround- 


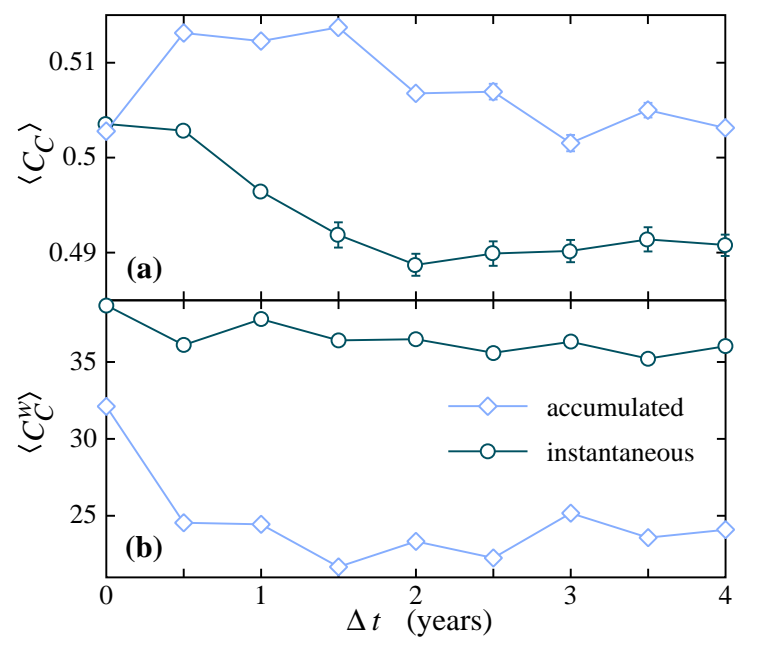

FIG. 4 Average closeness centralities of students who stay at the university nine semesters in total. (a) shows the unweighted version $d_{0}$ and (b) shows the weighted counterpart $d_{0}^{w}$.

ing. This section concerns a global centrality measure. The study of global centrality in graphs dates back to the 19th century (11). There are many different notions of centrality, and thus, many different measures, each trying to quantify a certain aspect of centrality (22). One of the simplest is the closeness centrality (3), the reciprocal average shortest distance to all other vertices:

$$
C_{C}(i)=\frac{N-1}{\sum_{j \neq i} d(i, j)} .
$$

(For weighted networks $d(i, j)$ is replaced by $d_{w}(i, j)$.) In Fig. 4 we plot $C_{C}$ and $C_{C}^{w}$ for the nine-semester students. The impression this figure gives us is a slowly decreasing centrality. Other lengths of the university stays are not less shaky, but all have a general downward trend. This leads us to the conclusion, that as one starts the university, taking general courses in big classes one is more central in the network of students than when one takes more specialized courses later in one's university education. It is worth noting that this holds even for the weighted accumulated networks-it does not matter that one gets closer to one's fellow freshmen (as discussed above), one still gets increasingly peripheral in the network as a whole. From the point of view of the educator, this analysis confirms what we already know: that students are formative mainly in the first semesters, when they are embedded in the short distances at the core of the course-affiliation network. After the first semesters, students' identities are already beginning to shape and you can merely add to the already given foundation. From that point it is unusual that students make dramatic shifts.

\section{Clustering coefficients}

Acquaintance networks are believed to have an overrepresentation of strongly connected triads $(8 ; 15)$. I.e., if A is strongly connected to B and C, then B and C are also likely to

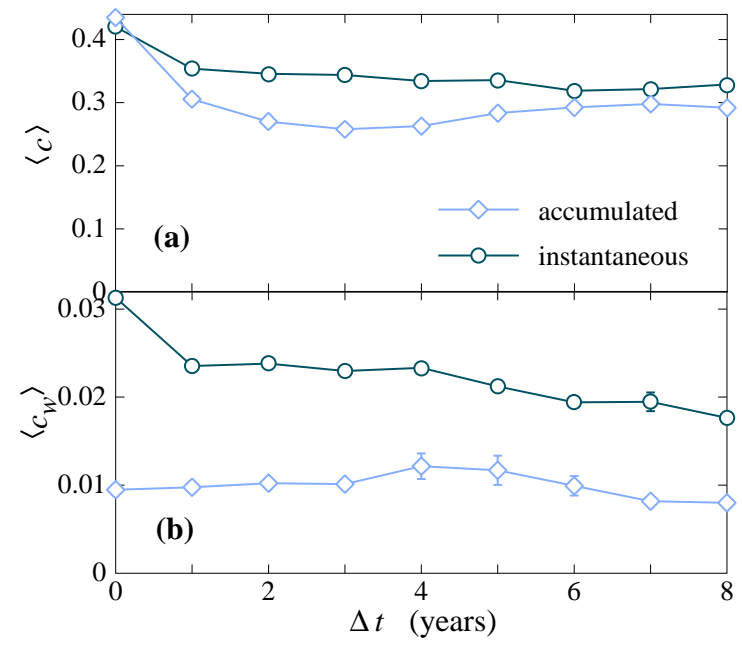

FIG. 5 Average clustering coefficient of students are a total of nine semesters at the University. (a) shows the unweighted version $\langle c\rangle$ and (b) shows the weighted counterpart $\left\langle c_{w}\right\rangle$. All errorbars are smaller than the symbol size.

have a strong connection. A quantity to measure the strength of connections within $i$ 's neighborhood is the local clustering coefficient (23):

$$
c(i)=e\left(\Gamma_{i}\right) /\left(\begin{array}{c}
k(i) \\
2
\end{array}\right),
$$

where $e\left(\Gamma_{i}\right)$ is the number of edges within $i$ 's neighborhood $\Gamma_{i}$. The time evolution of clustering coefficients for nine-semester students is plotted in Fig. [5] a). We believe the overall decrease reflects that the choice of courses a student take is increasingly specialized and individual (which of course decrease the fraction of fellow students themselves taking the same course).

The decreasing sizes of the classes as a student progress to more specialized subjects makes the weights between the students stronger. Consequently one can argue that the triads become stronger and the clustering coefficient should be adjusted to reflect this. It turns out that the generalization if the local clustering coefficient to weighted networks is not entirely trivial. We would like such a measure to fulfill the following requirements:

1. The values should lie in the interval $[0,1]$.

2. If the weight matrix is replaced by a 0,1 -adjacency matrix the Watts-Strogatz clustering coefficient should be recovered.

3. Zero weight should consistently represent the absence of an edge.

4. The contribution from one triad including the vertex $i$ to $i$ 's weighted clustering coefficient $c_{w}(i)$ to be proportional to the weight of each edge in the triad.

Unfortunately, none of the proposed definitions of a weighted clustering coefficients we are aware of $(2 ; 13 ; 18)$ fulfills all 


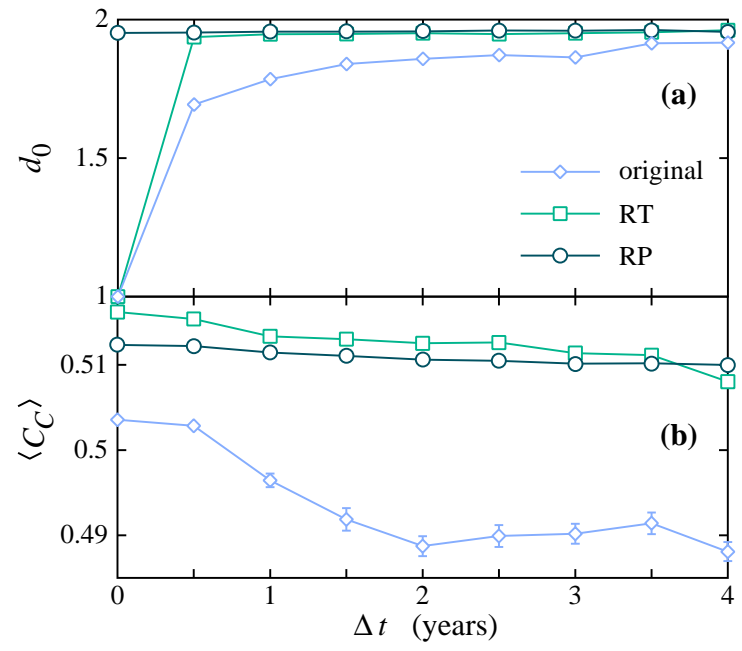

FIG. 6 Conditional uniform graph tests of $d_{0}$ (a) and $\left\langle C_{C}\right\rangle$ (b) for nine-semester students.

these requirements. We note that an alternative way of writing the Watts-Strogatz clustering coefficient is

$$
c(i)=\frac{\sum_{j k} a_{i j} a_{j k} a_{k i}}{\sum_{j k} a_{i j} a_{k i}}=\frac{\mathbf{A}_{i i}^{3}}{(\mathbf{A} \mathbf{1} \mathbf{A})_{i i}},
$$

where $\mathbf{1}$ is the matrix where all elements are 1. In this representation the generalization from an adjacency matrix to a weight matrix is straightforward

$$
c_{w}(i)=\frac{\sum_{j k} w_{i j} w_{j k} w_{k i}}{\max _{i j} w_{i j} \sum_{j k} w_{i j} w_{k i}}=\frac{\mathbf{W}_{i i}^{3}}{\left(\mathbf{W} \mathbf{W}_{\max } \mathbf{W}\right)_{i i}},
$$

where $\mathbf{W}_{\max }$ is the matrix with $\max _{i j} w_{i j}$ on all positions, which indeed fulfills all four requirements above. The weighted clustering coefficient counterparts of Fig.5]a) is displayed in Fig. 5 b). We see that, for the instantaneous networks, the increasing strength of the triads counterbalances the effect of more personalized curricula, so the $\left\langle C_{C}^{w}\right\rangle$ curves are flatter.

\section{CONDITIONAL UNIFORM GRAPH TESTS}

In this section we put some the results of the measurements in perspective by comparing them to results for graphs with some network structure averaged away. A thorough analysis of this kind, to establish the interpretations we propose above, would extend the size of the paper so much that we omit it. Instead we perform case studies of two of our unweighted quantities, $d_{0}$ and $\left\langle C_{C}\right\rangle$.

The standard way to view a complex network is to say it is to some extent random and that it also has some degree of structure-deviations from complete randomness induced by the forces forming the network $(7 ; 20)$. The commonly used structural measures are not independent. To sort out if a given quantity $X\left(G_{0}\right)$ (of a graph $G_{0}$ ) is a dependent on a certain other quantity $Y\left(G_{0}\right)$ one can perform a "conditional uniform graph test" $(12)$ and compare $X\left(G_{0}\right)$ with the value of $X$ averaged over graphs with $Y$ conditioned to $Y\left(G_{0}\right)$. In this paper we will perform two simple conditional uniform graph test: First we rewire the two-mode course-student network before making the projection to the student network, and constructing the weights (we call this RT randomization, mnemonic for "randomize two-mode"). We treat multiple course registrations as a result of the randomization as just one course registration. We also rewire the one-mode projections (RP, mnemonic for "randomize projection"). This gives Poisson random graphs of the same sizes as the real networks. For this networks we average the results over ten randomizations.

In Fig. 6a) we plot the average distance to the fellow freshmen for the RT and RP randomizations. We see that the randomized curves are approximately constant (apart from the RT curve where $d_{0}(0)=1$ by definition). The distances in the real networks are strictly shorter than in the randomized networks. This is completely consistent with a picture of students of similar subject being closer than students of different subjects-even if students are not classmates after a few semesters they are likely to take courses of similar subjects and thus be closer than an arbitrary other student.

Fig. 6 b) shows the closeness centrality of nine-semester students for real and randomized networks. We see that the students have considerably lower centrality values than the vertices of the test networks, and that the downward trend is stronger for the original values. That the closeness is on average smaller in the real networks reflects that the average distances are larger than in the randomized networks. This is logical if one assumes the student networks can be described as groups of students majoring in similar subjects. Both randomizations remove the tendency of students close to graduation take courses with few other students, which explains the stronger decline of the real-world curves. The downward trend of the randomized curves is explained by the increasing average distances of the random networks due to the increasing number of students.

\section{SUMMARY AND DISCUSSION}

We have analyzed a data set from a Korean university consisting of course registration lists for 18 semesters. These lists are made into weighted and unweighted networks of students. The weight between two students is a sum over all courses they have taken together. We argue that, if one wants the weight to represent the probability of an acquaintance along an edge, then the contribution to a weight from one particular course should be chosen as inversely proportional to the number of students taking the course. Furthermore, an old course should contribute less to an edge than a newer. We use two decay functions for the weights, one constant (minimally decreasing)_defining accumulated networks, and one zero for any course earlier than the present semester-defining instantaneous networks. An unweighted edge is defined to be present whenever the corresponding weight is non-zero. The quantities we measure, all in both weighted and unweighted versions, and all as function of the time a student has been 
present at the university are: Degree and closeness centralities; distances to fellow students of the first semester; and clustering coefficients. Some conclusions are strengthened by conditional uniform graph tests.

We find that students enter the university in the center of the student network, as a student progresses she, or he, will slowly become more peripheral. On the other hand, the ties with fellow students becomes stronger over time (to come to this conclusion one needs weights, but the decay-function does not matter). The connectedness of the neighborhood, as measured by the unweighted clustering coefficient, decreases with time. We argue that this should not be interpreted as the neighborhoods of students becomes weaker, also here the conclusion from the weighted quantity is more sound - that the triads of the instantaneous networks have roughly the same strength over time.

From a qualitative point of view, interpreting our courseregistration network, and affiliation networks in general, is problematic in the sense that it connects individuals with each other indirectly, and not through directly observed social interaction. This means we do not know if being in the same course also fosters significant social interaction or if it breeds friendship for example. From personal introspection only can we confirm that it sometimes did and it sometimes did not. Still, it is perfectly reasonable to assume that university course affiliation is an important identity shaper for a university student, and therefore the analysis bears considerable sociological relevance. In free societies most adult people join schools, clubs, organizations, etc. by choice. However, once affiliated one is under the influence of everything that goes on in that particular arena (25). At the university, most, but not all, of the students in a course are literary changing in front of our eyes in the duration of a course. Indeed, that is one of the rewards of teaching. And, once your students are finished with your course, they go on to other courses that have been chosen by them partly under the spell of whatever took place within the boundaries of that particular course you were offering. In this respect the analysis provides an illustration of the identity shaping processes that takes place, not only in university courses but in every social arena with which we are affiliated. Within the limits of course offerings, the network is shaped by this identity seeking on behalf of the students. Because individual identity is largely defined by exclusion of other identities $(4 ; 21)$ it is not surprising that we see a growing fragmentation and distance between students as time passes by. In network terms, university life as uncovered in the analysis, begins at the core and drifts to the periphery. In terms of identity shaping, the track is the opposite-students start off blank in scholarly identity and gradually shapes into quasi-experts of their majoring subject. And as the semesters pass, groups form around fellow students that are following the same identity path, i.e. students develop similar university identities.

\section{Acknowledgments}

We thank Ajou University for providing the course registration data and Mark Newman for comments. CRE thank
Wissenschaftskolleg zu Berlin. Financial support from Korea Research Foundation through Grant No. 2003-041-C00137 (BJK) and the Bank of Sweden Tercentenary Foundation (CRE) are gratefully acknowledged.

\section{References}

[1] Albert, R. and Barabási, A.-L. (2002). Statistical mechanics of complex networks. Rev. Mod. Phys, 74:47-98.

[2] Barrat, A., Barthélemy, M., Pastor-Santorras, R., and Vespignani, A. (2004). The architecture of complex weighted networks. Proc. Natl. Acad. Sci. USA, 101(11):3747-3752.

[3] Beauchamp, M. A. (1965). An improved index of centrality. Behav. Sci., 10:161-163.

[4] Bourdieu, P. (1984). Distinction: A social critique of the judgement of taste. Harvard University Press, Cambridge MA.

[5] Broder, A., Kumar, R., Maghoul, F., Raghavan, P., Rajagopalan, S., Stata, R., Tomkins, A., and Wiener, J. (2000). Graph structure in the web. Comput. Netw., 33:309-320.

[6] Dorogovtsev, S. N. and Mendes, J. F. F. (2003). Evolution of Networks: From Biological Nets to the Internet and WWW. Oxford University Press, Oxford.

[7] Fararo, T. J. and Sunshine, M. H. (1964). A study of a biased friendship net. Syracuse University Press, Syracuse, NY.

[8] Holland, P. W. and Leinhardt, S. (1972). Some evidence on the transitivity of positive interpersonal sentiment. Am. J. Sociol., 72:1205-1209.

[9] Holme, P. (2003). Network dynamics of ongoing social relationships. Europhys. Lett., 64(3):427-433.

[10] Holme, P., Edling, C. R., and Liljeros, F. (2004). Structure and time evolution of an Internet dating community. Social Networks, 26:155-174.

[11] Jordan, C. (1869). Sur les assemblages des lignes. Journal für die reine und angewandte Mathematik, 70:185-190.

[12] Katz, L. and Powell, J. H. (1957). Probability distributions of random variables associated with a structure of the sample space of sociometric investigations. Ann. Math. Stat., 28:442448.

[13] Li, M., Fan, Y., Chen, J., Gao, L., Di, Z., and Wu, J. Weighted networks of scientific communication: The measurement and geometrical role of weight. e-print cond-mat/0409272

[14] Luczkovich, J. J., Borgatti, S. P., Johnson, J. C., and Everett, M. G. (2003). Measuring trophic role similarity in food webs using regular equivalence. J. Theor. Biol., 220:303-321.

[15] Newcomb, T. M. (1961). The acquaintance process. Holt, Reinhart \& Winston, New York NY.

[16] Newman, M. E. J. (2001). Scientific collaboration networks. II. Shortest paths, weighted networks, and centrality. Phys. Rev. E, 64:016132.

[17] Newman, M. E. J. (2003). The structure and function of complex networks. SIAM Rev., 45:167-256.

[18] Onnela, J.-P., Saramäki, J., Kertész, J., and Kaski, K. Intensity and coherence of motifs in weighted complex networks. e-print cond-mat/0408629

[19] Park, S. M., Holme, P., and Kim, B. J. Student network in Ajou University based on the course registration data. (in Korean) to appear in Sae Mulli.

[20] Rapoport, A. (1957). Contribution to the theory of random and biased nets. Bull. Math. Biophysics, 19:257-277.

[21] Simmel, G. (1955). Conflict and the web of group-affiliations. Free Pres, Glencoe IL. 
[22] Wasserman, S. and Faust, K. (1994). Social network analysis: Methods and applications. Cambridge University Press, Cambridge.

[23] Watts, D. J. and Strogatz, S. H. (1998). Collective dynamics of 'small-world' networks. Nature, 393:440-442.
[24] Wellman, B. (2001). Computer networks as social networks. Science, 293:2031-2034.

[25] White, H. C. (1992). Identity and control: A structural theory of social action. Princeton University Press, Princeton. 